\title{
Arthroscopic Treatment for Femoroacetabular Impingement with Extraspinal Diffuse Idiopathic Skeletal Hyperostosis
}

Jung-Mo Hwang, MD, Deuk-Soo Hwang, MD, Chan Kang, MD, Woo-Yong Lee, MD, Gi-Soo Lee, MD, Jeong-Kil Lee, MD, Yun-Ki Kim, MD

Department of Orthopaedic Surgery, Chungnam National University School of Medicine, Daejeon, Korea

Background: Patients with extraspinal diffuse idiopathic skeletal hyperostosis (DISH) involving the hip joint have symptoms like femoroacetabular impingement (FAI). To date, no reported study has determined the clinical outcomes of arthroscopic treatment in extraspinal DISH involving the hip joint.

Methods: A total of 421 hips with FAl that underwent arthroscopic treatment were reviewed retrospectively. We determined the extraspinal involvement of DISH with three-dimensional computed tomography (3D-CT) and simple radiography of the pelvis and hip joint. Clinical outcomes were evaluated at a minimum of 2 years postoperatively. The visual analog scale score (VAS), modified Harris hip score (MHHS), and hip outcome score-activity of daily living scale (HOS-ADL) were used, and hip range of motion (ROM) was evaluated pre- and postoperatively and at the time of the final follow-up.

Results: Among the 421 hips (372 patients) with FAl that underwent arthroscopic treatment, 17 hips (12 patients, 4.04\%) had extraspinal DISH on the hip joints. The mean age of the patients was 51.5 years. The 3D-CT scans and simple radiographs showed extraspinal DISH on multiple points around the pelvis and hip joint. Nine of the 17 hips (seven of 12 patients) had spinal DISH. At the final follow-up, VAS, MHHS, and HOS-ADL improved significantly from 6.5, 65.3, and 66.6, respectively, to $1.2,87.8$, and 89.5, respectively, and hip flexion and internal rotation improved significantly from $97.7^{\circ}$ and $7.9^{\circ}$, respectively, to $117.1^{\circ}$ and $18.2^{\circ}$, respectively.

Conclusions: This study has demonstrated that extraspinal DISH involving the hip joint could lead to FAI, and arthroscopic treatment could result in relief of symptoms, including pain and ROM limitation, in extraspinal DISH patients.

Keywords: Arthroscopy, Hip, Diffuse idiopathic skeletal hyperostosis

Diffuse idiopathic skeletal hyperostosis (DISH) is a condition characterized by abnormal calcification and ossification of ligaments and entheseal sites. ${ }^{1}$ In 1950, this condition, which was termed senile ankylosing hyperostosis, was first reported by Forestier and Rotes-querol ${ }^{2)}$ who described the radiological aspects of the condition with its

Received February 19, 2019; Accepted April 8, 2019

Correspondence to: Deuk-Soo Hwang, MD

Department of Orthopaedic Surgery, Chungnam National University

School of Medicine, 266 Munhwa-ro, Jung-Gu, Daejeon 35015, Korea

Tel: +82-42-338-2480, Fax: +82-42-338-2482

E-mail: dshwang@cnu.ac.kr predilection to the thoracic spine but also to the lumbar and cervical spine. The axial skeleton is often involved, particularly the thoracic spine, but involvement of peripheral or appendicular skeleton led researchers to use the name DISH. ${ }^{3,4)}$

The diagnostic criteria most commonly used for DISH were proposed by Resnick and Niwayama ${ }^{3)}$ in 1976 and required flowing anterolateral ossifications of at least four contiguous thoracic vertebral segments, preservation of the intervertebral disc spaces, and absence of apophyseal joint degeneration or sacroiliac inflammatory changes. However, many reports have stated that DISH affects extraspinal locations and is not limited to the spinal 
Hwang et al. Hip Arthroscopy for Extraspinal Diffuse Idiopathic Skeletal Hyperostosis

Clinics in Orthopedic Surgery • Vol. 11, No. 3, 2019•www.ecios.org

column and suggested that the radiographic appearance in the peripheral skeleton might be characteristic enough to suggest the diagnosis of DISH, even in the absence of axial skeleton radiographs. ${ }^{5-8)}$ As a result, in 1985, another set of criteria, defined by Utsinger ${ }^{9)}$ as probable DISH, meaning maybe the early stage of DISH, lowered the threshold for spinal involvement to at least three adjacent vertebral bodies, but added the presence of peripheral enthesopathies to the diagnostic measures. Many extraspinal entheseal sites-such as the pelvis, tibia, patella, calcaneus, and olecranon-could be involved. ${ }^{5-10)}$ Therefore, if extraspinal DISH involves the hip joint, particularly the superior acetabulum, hip pain and range of motion (ROM) limitation like femoroacetabular impingement (FAI) symptoms could be present. ${ }^{11,12)}$

We hypothesized that extraspinal DISH involving the hip joint, particularly the periacetabular site, could lead to FAI symptoms and that arthroscopic treatment, such as arthroscopic decompression of hyperostosis of the acetabulum, could result in patient satisfaction and improved clinical outcomes. To our knowledge, few studies have reviewed extraspinal DISH. In addition, no reported study has determined the clinical outcomes of arthroscopic treatment in extraspinal DISH involving the hip joint. The purpose of this study was to determine clinical outcomes in patients with extraspinal DISH who underwent arthroscopic treatment of the hip joint.

\section{METHODS}

\section{Patient Selection}

From January 2007 to January 2012, 421 hips of 372 patients with FAI who underwent arthroscopic treatment at Chungnam National University Hospital were reviewed retrospectively. All of the hips underwent three-dimensional computed tomography (3D-CT) of the hip. Based on the radiographic assessment of the acetabular index angle, lateral center-edge angle, alpha angle, and the presence of acetabular retroversion, FAI was classified as cam, pincer, or mixed type. Also, we determined the extraspinal involvement of DISH using the 3D-CT scans and simple radiography of the pelvis and hip joint. We identified the points of extraspinal DISH on the pelvis and hip joint according to the 14 pelvic points suggested by Haller et al.,5) which are symphysis pubis, iliopubic line, sacrotuberous ligament, inferior sacroiliac joint, superior sacroiliac joint, iliolumbar ligament, iliac crest, iliac wing, superior acetabulum, inferior acetabulum, greater trochanter, lesser trochanter, obturator foramen, and ischial tuberosity. All cases had insidious onset of hip pain or pain that followed low-energy trauma and positive hip impingement signs. ${ }^{13)}$ In particular, the anterior hip impingement test was usually positive. Therefore, the inclusion criteria were the case with following conditions: ROM limitation of the hip joint, particularly flexion and internal rotation, positive hip impingement sign, and peripheral enthesopathy or hyperostosis on the periacetabular site with sparing of the joint space and surface in simple radiography (Fig. 1) and 3D-CT of the hip joint. ${ }^{8,11}$ Cases with the following were excluded: a history of high-energy trauma to the hip (i.e., fracture or dislocation), a history of surgery involving the hip or pelvis, Tönnis grade 2 or above, ${ }^{14)}$ proliferative disease of the hip (i.e., synovial chondromatosis or pigmented villonodular synovitis), neuromuscular disease (i.e., cerebral palsy), Legg-Calvé-Perthes deformity, the peripheral type of ankylosing spondylitis, or developmental dysplasia of the hip. All of the patients provided informed consent and Institutional Review Board approval was obtained (IRB No. CNUH 2015-06-020).

\section{Surgical Technique and Postoperative Care}

All operations were performed by a single surgeon, the
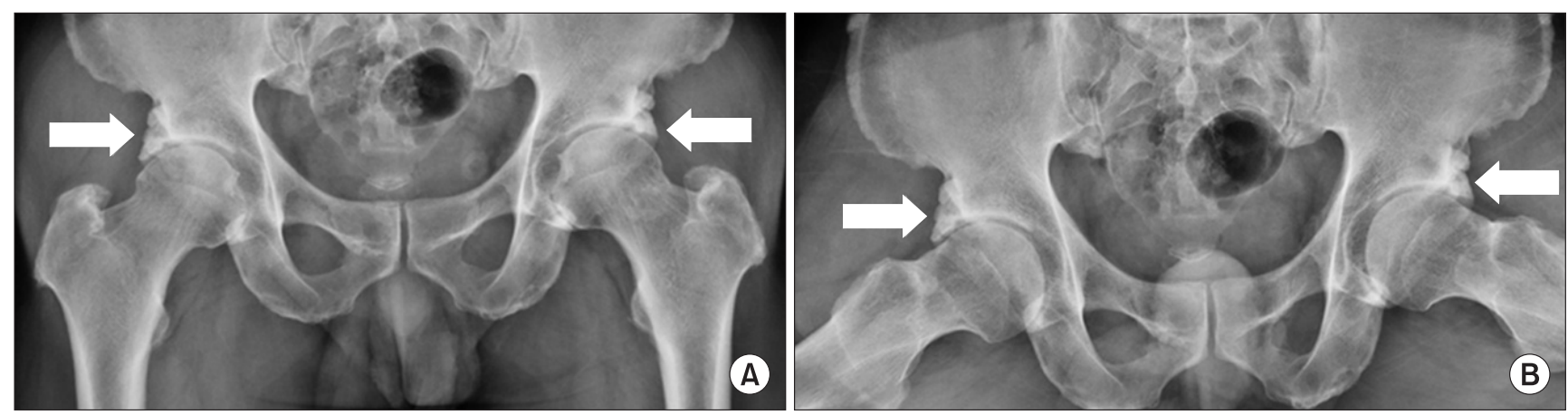

Fig. 1. Preoperative simple anteroposterior $(A)$ and frog-leg $(B)$ radiographs of the hip joint showing hyperostosis on the anterosuperior acetabular rim (arrows) of both sides with sparing of the joint space and surface. 
Hwang et al. Hip Arthroscopy for Extraspinal Diffuse Idiopathic Skeletal Hyperostosis

Clinics in Orthopedic Surgery • Vol. 11, No. 3, 2019• www.ecios.org

senior author (DSH), with the patient under general anesthesia. Hip arthroscopy was performed with the patient in the supine position on a standard hip traction table, and the operative limb was placed in slight hip flexion, abduction relative to the pelvis, and $15^{\circ}$ to $20^{\circ}$ of hip internal rotation with traction. The contralateral limb was positioned in extension and neutral rotation, with the foot placed in a support with application of a counterbalancing traction. Countertraction, lateralized toward the operative hip, was placed in the perineal region. Anterior, anterolateral, and sometimes posterolateral or medial portals were used. ${ }^{15-17)}$ The central compartment was addressed first. Concomitant lesions (labral tear, ligamentum teres injury, or pincer impingement, etc.) were evaluated and eventually treated. After central compartment manipulation, the traction was removed and the hip was flexed about $40^{\circ}$. Attention was focused on the periacetabular site under the anterior inferior iliac spine (AIIS). Arthroscopic shaver and radiofrequency ablation probe were used to remove all soft tissue from the protruded acetabulum and to better demarcate the plane between the acetabular rim and the AIIS. Then, a burr was used to decompress hyperostosis on the peri acetabular site under the AIIS to conserve the indirect head of the rectus femoris footprint (Fig. 2). In addition, femoroplasty was performed when cam deformity was found by preoperative radiographic assessment. After decompression of hyperostosis on the periacetabular site and/or prominence at the femoral head-neck junction, we identified whether impingement during dynamic hip motion with flexion and rotation was present or not. Postoperatively, under general anesthesia, passive motions of the hip joint were identified for comparison with preoperative hip motion in patients with ROM limitation.

All cases underwent 3D-CT scans at 2 days after operation to check the postoperative state of the decompression, acetabuloplasty, and femoroplasty (Fig. 3). The patient consented to undergoing the postoperative 3D-CT scans before the surgery. Partial weight-bearing ambula-
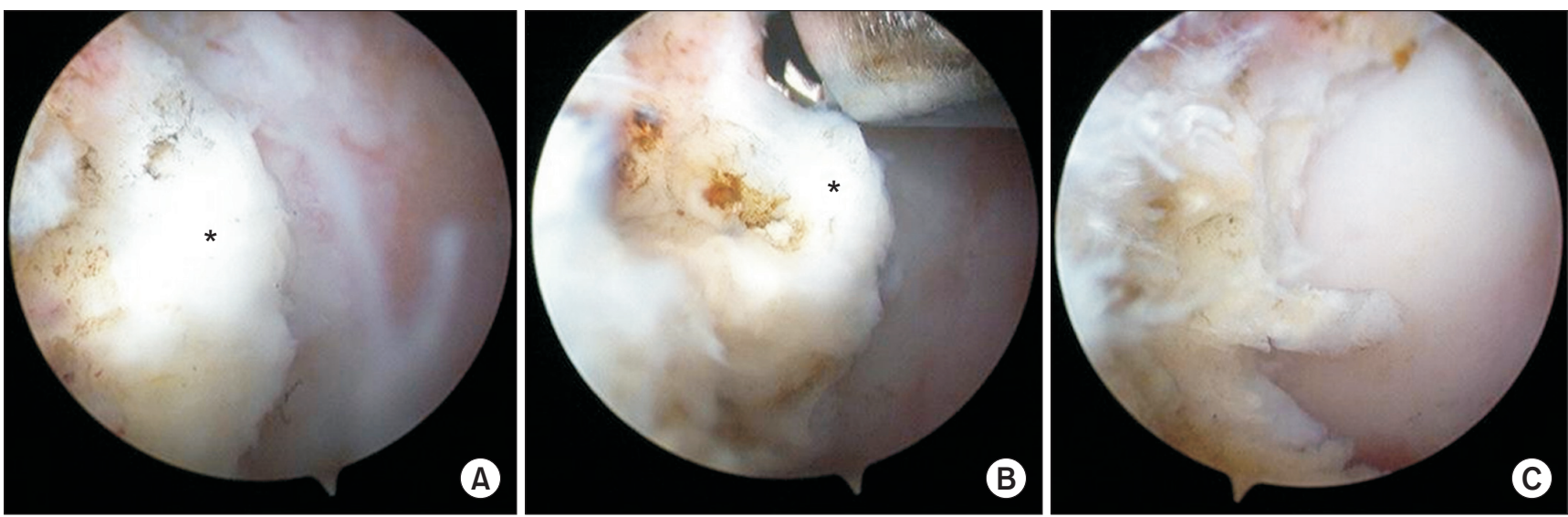

Fig. 2. Arthroscopic views from the anterolateral portal. (A) Hyperostosis on the anterosuperior side of the acetabular rim (asterisk). (B) Decompression of hyperostosis using a burr (asterisk). (C) After decompression of hyperostosis.
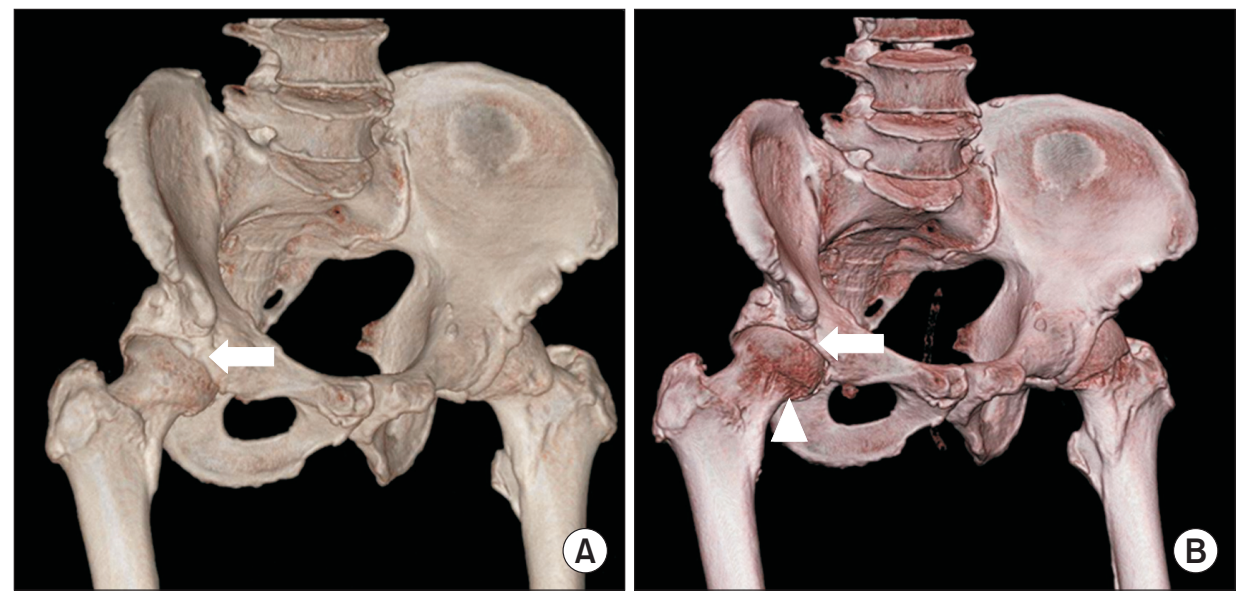

Fig. 3. (A) Preoperative three-dimensional computed tomography (3D-CT) view of the hip joint in $30^{\circ}$ internal rotation showing mixed-type femoroacetabular impingement and hyperostosis (arrow) on the anterosuperior acetabulum below the anterior inferior iliac spine. (B) Postoperative 3D-CT view of the hip joint in $30^{\circ}$ internal rotation showing the results of femoroplasty (arrowhead) and acetabuloplasty with decompression of hyperostosis (arrow). 
Hwang et al. Hip Arthroscopy for Extraspinal Diffuse Idiopathic Skeletal Hyperostosis

Clinics in Orthopedic Surgery • Vol. 11, No. 3, 2019• www.ecios.org

tion with crutches was maintained until 4 weeks after surgery in patients who had undergone osteoplasty and labral repair. Pendulum exercise and continuous passive motion were encouraged after the procedure to avoid postoperative capsular adhesion. ${ }^{18)}$ For preventing postoperative infection, antibiotics were ordered for 3 days and for control of pain and heterotrophic ossification, nonsteroidal antiinflammatory drugs were ordered for 3-4 weeks.

\section{Clinical Evaluation}

The clinical outcomes were evaluated at a minimum of 2 years postoperatively. The visual analog scale (VAS) score, modified Harris hip score (MHHS), ${ }^{199}$ and hip outcome score-activity of daily living scale (HOS-ADL) ${ }^{20)}$ were used. Hip ROM was assessed as part of clinical evaluations before surgery and at the final follow-up.

\section{Statistical Analysis}

Differences between preoperative and final follow-up clinical outcomes were analyzed with the paired $t$-test. IBM SPSS ver. 19.0 (IBM Corp., Armonk, NY, USA) was used. A difference was considered to be statistically significant at $p$-values $<0.05$.

\section{RESULTS}

Only 17 hips (4.04\%, 12 patients) among 421 hips (372 patients) were determined to have extraspinal DISH involving the hip joint. There were nine males and three females. The mean age of patients and follow-up period of the hips were 51.5 years (range, 39 to 70 years) and 43.6 months (range, 24 to 84 months), respectively. All of the patients had inguinal pain with ROM limitation. All of the patients had extraspinal DISH on multiple points around the pelvis and hip joint (at least 5 points) and had inferior sacroiliac joint, superior acetabulum, and ischial tuberosity points (Table 1 ). Nine of the 17 hips (58.33\%, seven of 12 patients) had spinal DISH. Among the 17 hips assessed in the present study, 0 hip, three hips (17.6\%), and 14 hips (82.4\%) were categorized as cam, pincer, and mixed type, respectively (Table 1). At the final follow-up, VAS, MHHS, and HOS-ADL improved significantly from 6.5, 65.3, and 66.6 , respectively, to $1.2,87.8$, and 89.5 , respectively, and hip flexion and internal rotation improved significantly from $97.7^{\circ}$ and $7.9^{\circ}$, respectively, to $117.1^{\circ}$ and $18.2^{\circ}$, respectively (all $p<0.001$ ) (Table 2 ). There were no intra- or perioperative complications. No patient had neural injury, wound infection, or avulsion of the indirect head of rectus femoris origin.

\section{Table 1. Demographics of 12 Patients with Extraspinal DISH of the Hip Joint}

\begin{tabular}{|c|c|c|c|c|c|c|c|c|}
\hline $\begin{array}{c}\text { Age at } \\
\text { surgery (yr) }\end{array}$ & Sex & $\begin{array}{l}\text { Affected } \\
\text { hip }\end{array}$ & Pelvic-point of DISH* & $\begin{array}{c}\text { Spinal } \\
\text { DISH }\end{array}$ & FAl type & Cause of injury & Onset (yr) & $\underset{\left(\mathrm{kg} / \mathrm{m}^{2}\right)}{\mathrm{BMI}}$ \\
\hline 59 & Male & Right & $3,4,6,7,8,9,11,12,14$ & No & Pincer & None & 0.5 & 28.7 \\
\hline 59 & Male & Left & $3,4,5,6,7,8,9,10,11,14$ & Yes & Mixed & Slip down & 2 & 24.2 \\
\hline 70 & Male & Right & $2,3,4,5,6,7,8,9,10,11,12,14$ & Yes & Mixed & None & 5 & 23.9 \\
\hline 42 & Male & Left & $1,2,3,4,5,6,7,8,9,10,12,13,14$ & Yes & Mixed & None & 10 & 20.0 \\
\hline $43-44$ & Male & Both & $3,4,6,7,8,9,10,11,12,14$ & No & Mixed & None & 3 & 26.6 \\
\hline $59-60$ & Male & Both & $3,4,7,9,14$ & No & Pincer & Slip down & 0.5 & 19.3 \\
\hline $41-42$ & Female & Both & $4,5,6,7,8,9,10,14$ & No & Mixed & None & 8 & 28.7 \\
\hline $39-40$ & Female & Both & $1,3,4,7,9,10,11,13,14$ & Yes & Mixed & None & 2 & 32.1 \\
\hline $69-70$ & Female & Both & $3,4,6,7,8,9,10,11,12,14$ & Yes & Mixed & None & 2 & 25.4 \\
\hline 50 & Male & Right & $1,3,4,5,6,7,8,9,11,12,14$ & Yes & Mixed & None & 3 & 24.2 \\
\hline 41 & Male & Left & $2,3,4,5,6,7,8,9,11,12,14$ & No & Mixed & None & 0.5 & 26.7 \\
\hline 47 & Male & Left & $4,6,9,13,14$ & Yes & Mixed & Stretching exercise & 1 & 24.1 \\
\hline
\end{tabular}

DISH: diffuse idiopathic skeletal hyperostosis, FAl: femoroacetabular impingement, BMI: body mass index.

*1: symphysis pubis, 2: iliopubic line, 3: sacrotuberous ligament, 4: inferior sacroiliac joint, 5: superior sacroiliac joint, 6: iliolumbar ligament, 7: iliac crest, 8: iliac wing, 9: superior acetabulum, 10: inferior acetabulum, 11: greater trochanter, 12: lesser trochanter, 13: obturator foramen, 14: ischial tuberosity. 
Hwang et al. Hip Arthroscopy for Extraspinal Diffuse Idiopathic Skeletal Hyperostosis

Clinics in Orthopedic Surgery • Vol. 11, No. 3, 2019•www.ecios.org

Table 2. Comparison of the Preoperative State and Clinical Outcome at the Final Follow-up

\begin{tabular}{lccc}
\multicolumn{1}{c}{ Outcome } & Preoperative & Final follow-up & $p$-value ${ }^{*}$ \\
VAS & $6.47 \pm 0.72$ & $1.18 \pm 0.88$ & $<0.001$ \\
MHHS & $65.29 \pm 7.66$ & $87.81 \pm 2.56$ & $<0.001$ \\
HOS-ADL & $66.60 \pm 7.22$ & $89.51 \pm 3.65$ & $<0.001$ \\
Flexion $\left(^{\circ}\right)$ & $97.65 \pm 4.72$ & $117.06 \pm 8.49$ & $<0.001$ \\
\hline Internal rotation $\left(^{\circ}\right)$ & $7.94 \pm 3.56$ & $18.24 \pm 3.51$ & $<0.001$ \\
\hline
\end{tabular}

Values are presented as mean \pm standard deviation.

VAS: visual analog scale score, MHHS: modified Harris hip score, HOS-

ADL: hip outcome score-activity of daily living scale.

*Based on separate paired $t$-test, $p<0.05$ denotes statistical significance.

\section{DISCUSSION}

The principal findings of this study are as follows: (1) New diagnostic criteria of DISH involving the peripheral site should be developed because extraspinal DISH could be present in various sites throughout the body without evidence of spinal DISH, abnormalities of pelvic bone were the most common. (2) Hip arthroscopy should be considered a good indication for patients with FAI symptoms and ROM limitation due to extraspinal DISH.

DISH is a noninflammatory disease of unknown etiology characterized by calcification and ossification of soft tissues, such as ligaments, tendons, and fascia. ${ }^{21)}$ The main target of the disease process is within the enthesis, an organ rich in collagen fibers, fibroblasts, and other mesenchymal cells, fibrocartilage, and calcified matrix that penetrates the bone cortex at its attachment. ${ }^{22)}$ The prevalence increases with age and has been reported to be $2.8 \%$ and $2.6 \%$ in males and females aged over 40 years, respectively. $^{23)}$

In 1950, Forestier and Rotes-Querol ${ }^{2)}$ described the radiologic, clinical, and pathologic features arising from nine cases and two autopsies of patients with senile ankylosing hyperostosis of the spine, and thereby rediscovered a clinical entity originally described in the 19th century. Therefore, 118 years have passed since DISH was introduced; however, the disorder remains poorly understood. ${ }^{7)}$ Although mechanical, environmental, genetic, metabolic, and endocrine factors have been examined in attempts to explain its etiology, the cause of DISH remains unknown. ${ }^{4,724)}$ Also, the diagnostic criteria of DISH are ambiguous. Even though the diagnostic criteria most commonly used for DISH were proposed by Resnick and Niwayama $^{3)}$ in 1976, extraspinal involvement was ignored. In
1985, another set of criteria, defined by Utsinger ${ }^{9)}$ as probable DISH, meaning maybe the early stage, lowered the threshold for spinal involvement to at least two contiguous vertebral bodies, but added the presence of peripheral enthesopathies involving the posterior heel, superior patella, or olecranon to the diagnostic measures. However, these criteria ignored other peripheral sites, such as the pelvis including the hip, the femur, and the hand including the wrist. $^{5-10)}$ This study showed that five of 12 patients had only extraspinal DISH with no evidence of spinal DISH. Therefore, new criteria of DISH including peripheral site should be developed.

Peripheral involvement in DISH is characterized by several distinctive features, including involvement of joints usually unaffected by primary osteoarthritis (OA), increased hypertrophic changes compared with primary OA, prominent enthesopathies at various sites adjacent to peripheral joints, and calcification and ossification of entheses in sites other than joints. ${ }^{11)}$ The main clinical manifestations of extraspinal DISH are pain and ROM limitation. ${ }^{1)}$ Although Utsinger ${ }^{9}$ added peripheral enthesopathies involving the posterior heel, superior patella, or olecranon to the diagnostic measures for probable DISH, extraspinal DISH could be involved in various sites throughout the body. ${ }^{5-10)}$ Among the extraspinal radiographic manifestations of DISH, abnormalities of the pelvic bone are most common. ${ }^{8,24)}$ Such pelvic abnormalities consist of bone proliferation, ligamentous calcification and ossification, and periarticular osteophytes. ${ }^{2,25-27)}$ Ossification with bony overgrowth of the pelvis including the hip joint has a particular predictive value for the presence of DISH. ${ }^{5,6,8)}$ In this study, five of 12 patients without spinal DISH were in an early stage of DISH from the vantage of probable DISH, and spinal DISH may be identified in them in the future. Therefore, a long-term follow-up will be necessary to identify.

In 1975, Resnick et al. ${ }^{8)}$ reported that among 21 consecutive patients with ankylosing hyperostosis of the spine, radiographic abnormalities of the pelvis were demonstrated in all 21 patients including the acetabula in seven patients, and five patients showed diminishing ROM in the hips. In the same manner, 12 patients in this study had radiologic abnormalities on multiple points of the pelvis and ROM limitations. Clinical outcomes including VAS, MHHS, HOS-ADL, and ROM significantly improved after arthroscopic treatment in these 12 patients.

This study has several limitations. First, we could not confirm that the extraspinal DISH was the early stage of DISH. Therefore, a long-term follow-up will be necessary. Second, we could not design a prospective compara- 
tive study due to lack of patients with extraspinal DISH involving the hip and FAI symptoms. Nevertheless, there was also a strength to this study. To our knowledge, this is the first clinical study of extraspinal DISH involving the hip joint causing FAI symptoms. Most other reports of extraspinal DISH were radiologic studies, reviews, and editorials.

Extraspinal DISH involving the hip joint could lead to FAI, and arthroscopic treatment could result in relief of symptoms, including pain and ROM limitation, in extraspinal DISH patients. In addition, among patients with FAI, extraspinal DISH involving the hip joint should be considered a cause of FAI in adults, and patients with
FAI who have painful hip motion and limitation of ROM should be evaluated to determine whether they have extraspinal DISH involving the hip.

\section{CONFLICT OF INTEREST}

No potential conflict of interest relevant to this article was reported.

\section{ACKNOWLEDGEMENTS}

This work was supported by research fund of Chungnam National University.

\section{REFERENCES}

1. Mader R, Buskila D, Verlaan JJ, et al. Developing new classification criteria for diffuse idiopathic skeletal hyperostosis: back to square one. Rheumatology (Oxford). 2013;52(2):326-30.

2. Forestier J, Rotes-Querol J. Senile ankylosing hyperostosis of the spine. Ann Rheum Dis. 1950;9(4):321-30.

3. Resnick D, Niwayama G. Radiographic and pathologic features of spinal involvement in diffuse idiopathic skeletal hyperostosis (DISH). Radiology. 1976;119(3):559-68.

4. Utsinger PD, Resnick D, Shapiro R. Diffuse skeletal abnormalities in Forestier disease. Arch Intern Med. 1976;136(7):763-8.

5. Haller J, Resnick D, Miller CW, et al. Diffuse idiopathic skeletal hyperostosis: diagnostic significance of radiographic abnormalities of the pelvis. Radiology. 1989;172(3):835-9.

6. Littlejohn GO, Urowitz MB. Peripheral enthesopathy in diffuse idiopathic skeletal hyperostosis (DISH): a radiologic study. J Rheumatol. 1982;9(4):568-72.

7. Mata S, Fortin PR, Fitzcharles MA, et al. A controlled study of diffuse idiopathic skeletal hyperostosis: clinical features and functional status. Medicine (Baltimore). 1997;76(2):10417.

8. Resnick D, Shaul SR, Robins JM. Diffuse idiopathic skeletal hyperostosis (DISH): Forestier's disease with extraspinal manifestations. Radiology. 1975;115(3):513-24.

9. Utsinger PD. Diffuse idiopathic skeletal hyperostosis. Clin Rheum Dis. 1985;11(2):325-51.

10. Sarzi-Puttini P, Atzeni F. New developments in our understanding of DISH (diffuse idiopathic skeletal hyperostosis). Curr Opin Rheumatol. 2004;16(3):287-92.

11. Mader R, Sarzi-Puttini P, Atzeni F, et al. Extraspinal mani- festations of diffuse idiopathic skeletal hyperostosis. Rheumatology (Oxford). 2009;48(12):1478-81.

12. Terzi R. Extraskeletal symptoms and comorbidities of diffuse idiopathic skeletal hyperostosis. World J Clin Cases. 2014;2(9):422-5.

13. Klaue K, Durnin CW, Ganz R. The acetabular rim syndrome: a clinical presentation of dysplasia of the hip. J Bone Joint Surg Br. 1991;73(3):423-9.

14. Tonnis D. Normal values of the hip joint for the evaluation of X-rays in children and adults. Clin Orthop Relat Res. 1976;(119):39-47.

15. Glick JM, Sampson TG, Gordon RB, Behr JT, Schmidt E. Hip arthroscopy by the lateral approach. Arthroscopy. 1987;3(1):4-12.

16. Ide T, Akamatsu N, Nakajima I. Arthroscopic surgery of the hip joint. Arthroscopy. 1991;7(2):204-11.

17. Kang C, Hwang DS, Hwang JM, Park EJ. Usefulness of the medial portal during hip arthroscopy. Clin Orthop Surg. 2015;7(3):392-5.

18. Philippon M, Schenker M, Briggs K, Kuppersmith D. Femoroacetabular impingement in 45 professional athletes: associated pathologies and return to sport following arthroscopic decompression. Knee Surg Sports Traumatol Arthrosc. 2007;15(7):908-14.

19. Mahomed NN, Arndt DC, McGrory BJ, Harris WH. The Harris hip score: comparison of patient self-report with surgeon assessment. J Arthroplasty. 2001;16(5):575-80.

20. Martin RL, Philippon MJ. Evidence of validity for the hip outcome score in hip arthroscopy. Arthroscopy. 2007;23(8):822-6.

21. Holton KF, Denard PJ, Yoo JU, et al. Diffuse idiopathic 
Hwang et al. Hip Arthroscopy for Extraspinal Diffuse Idiopathic Skeletal Hyperostosis

Clinics in Orthopedic Surgery • Vol. 11, No. 3, 2019• www.ecios.org

skeletal hyperostosis and its relation to back pain among older men: the MrOS Study. Semin Arthritis Rheum. 2011;41(2):131-8.

22. Mader R. Diffuse idiopathic skeletal hyperostosis: time for a change. J Rheumatol. 2008;35(3):377-9.

23. Julkunen H, Heinonen OP, Knekt P, Maatela J. The epidemiology of hyperostosis of the spine together with its symptoms and related mortality in a general population. Scand J Rheumatol. 1975;4(1):23-7.

24. Resnick D, Shapiro RF, Wiesner KB, Niwayama G, Utsinger PD, Shaul SR. Diffuse idiopathic skeletal hyperostosis
(DISH) [ankylosing hyperostosis of Forestier and RotesQuerol]. Semin Arthritis Rheum. 1978;7(3):153-87.

25. Forestier J, Lagier R. Ankylosing hyperostosis of the spine. Clin Orthop Relat Res. 1971;74:65-83.

26. Forestier J, Lagier R, Certonciny A. Concept of vertebral ankylosing hyperostosis: anatomo-radiological approach. Rev Rhum Mal Osteoartic. 1969;36(12):655-61.

27. Harris J, Carter AR, Glick EN, Storey GO. Ankylosing hyperostosis, I: clinical and radiological features. Ann Rheum Dis. 1974;33(3):210-5. 